\title{
AU FIL DE LA REVUE INTERNATIONALE DE SYSTEMIQUE : LES CONCEPTS DE LA SYSTÉMIQUE APPLIQUÉS À LA GESTION DES CRISES
}

\author{
Author(s) / Auteur(s) : \\ Gérard DONNADIEU \\ Vice-Président de l'AFSCET \\ Professeur de sciences religieuses aux Facultés jésuites de Paris et au Collège des Bernardins \\ gerard.donnadieu@wanadoo.fr
}

\begin{abstract}
Résumé :
Au cours de ses douze années d'existence, la Revue Internationale de Systémique (RIS) a publié plusieurs articles de fond, de nature épistémologique, sur l'approche systémique et ses concepts. L'auteur de cette communication se propose de reprendre brièvement les plus importants de ces articles afin de rechercher s'ils font apparaître une évolution dans la définition et le traitement d'un certain nombre de concepts clefs de la systémique, en particulier ceux relatifs à la gestion des crises, aux problèmes de conduite du changement, aux phénomènes d'émergence. Par exemple, observe-t-on au cours de ces douze ans, un approfondissement, une simple continuité logique ou une rupture paradigmatique dans la manière d'aborder ces notions? Et cela aussi bien au niveau des systèmes artificiels, des systèmes vivants et des systèmes humains et sociaux?
\end{abstract}

Keywords / Mots-clés :

ago-antagonisme, autonomie, auto-organisation, complexité, évolution, émergence, hétéro-organisation, systémique

La Revue Internationale de Systémique, au cours de ses douze années d'existence, a été un lieu privilégié de débats pour la communauté des systémiciens, en particulier de langue française. Il nous a semblé intéressant et utile de procéder à un survol rétrospectif des 300 articles environ qui y furent publié de manière à y retrouver les moments forts de la réflexion, les défis du moment, l'émergence de nouveaux concepts, avec une attention particulière à tout ce qui concerne les problèmes de changement, la gestion des crises, la prise en compte de la nouveauté.

Dans l'impossibilité de procéder à une étude comparative et synthétique de l'ensemble de ces articles, nous en avons retenu quatre à titre d'exemples nous ayant paru révélateurs des problématiques de la période et porteurs en même temps de nouveauté.

\section{LAMARCK ET L'ANALOGIE BIOLOGIQUE EN ECONOMIE}

(Ragip Ege, 1993 : vol.7, n5, pp.471-486)

L'économiste du changement technique trouve dans le modèle de l'évolution de Lamarck un certain nombre de concepts qui semblent plus appropriés que les concepts darwiniens pour l'analyse des processus et d'innovation en économie. L'auteur montre que l'analyse de l'action humaine, laquelle intègre les notions essentielles d'intentionnalité et de projet ne peut se réduire au mécanisme aveugle de la sélection darwinienne. En termes schumpétériens, la question que se pose l'économiste de l'innovation concerne les conditions de possibilité de l'apparition de la nouveauté dans un système économique marqué principalement par l'équilibre des "flux circulaires". Pour une telle tâche, certains concepts lamarckiens qui confèrent un rôle actif et interventionniste à l'individu semblent bien plus appropriés. 
Abandonnée par la plupart des biologistes qui en faisant le choix de la "théorie synthétique de l'évolution" lui ont préféré la référence à Darwin, la pensée de Lamarck ferait -elle ainsi un comeback révélateur grâce à sa récupération par les économistes ? L'auteur écrit : "L'intérêt porté aux rapports et à l'organisation rend Lamarck attentif au fait que l'être vivant n'est pas une structure isolée dans le vide, mais qu'il s'insère dans la nature avec laquelle il joue des relations variées. L'organisme doit réagir aux circonstances extérieures, et cette réaction constitue fondamentalement le moteur de l'évolution. Chez Lamarck, le concept d'individu possède un statut théorique sans commune mesure avec ce qu'on observe dans les analyses de Darwin. Alors que l'action du vivant est considérée comme la source fondamentale des variations chez Lamarck". Et il ajoute "qu'il s'agit là de la logique d'un processus d'adaptation et d'apprentissage, certes considérablement lent dans le temps mais résolu, qui ne pouvait pas ne pas retenir l'attention de l'économiste".

Ainsi l'agent économique, bien loin d'être soumis passivement aux lois du marché, dispose d'une large marge d'autonomie qui lui permet "d'inventer" des réponses nouvelles aux évolutions de son environnement. François Perroux parlera "d'unité active" pour qualifier cette créativité de l'acteur économique qui le rapproche de l'autonomie de l'être vivant dans le modèle lamarckien.

Faut-il déduire de cette analyse que l'analogie biologique est impropre pour expliquer le social et l'économique ? L'auteur n'est pas loin de le penser. Les deux domaines auraient leurs lois propres, aucune analogie n'étant entre eux possible.

Mais on peut aujourd'hui se reconnaitre sur une autre réponse, alors que la théorie synthétique de l'évolution est en crise et que le modèle néo-darwinien prend eau de toute part. Remettre en question ce modèle darwinien dans la biologie elle-même en faisant retour à un lamarckisme rénové, prenant appui par exemple sur les nouveaux travaux de l'épi-génétique et de l'embryologie. L'analogie économie/biologie se trouve alors à nouveau validée et avec elle une certaine vision de l'unité de la science.

\section{REEVALUATION DU CONCEPT D'EMERGENCE A LA LUMIERE DE LA SYSTEMIQUE AGO-ANTAGONISTE}

(Elie Bernard-Weil, 1994 : vol.8, n³, pp.315-335)

Comme l'a mis en évidence le systémicien américain Mario Bunge, les systèmes complexes se caractérisent par un empilement de niveaux d'organisation avec à chaque niveau l'apparition de propriétés nouvelles que l'on qualifie du terme mystérieux d'émergence et que l'on va imputer à la capacité d'auto-organisation de ces systèmes. Dans le processus d'émergence, le niveau sus-jacent, celui qui apparaît, est à la fois déterminé et non déterminé par le niveau sous-jacent. Certes il est "éclaboussé" par le niveau sous-jacent, note E. Bernard-Weil, "puisque l'existence de ce dernier est la condition d'apparition du niveau supérieur, mais en même temps le niveau sous-jacent s'efface en quelque sorte devant la complexité accrue du niveau émergent." Mais pourquoi un niveau émergeraitil ? Ainsi après l'avoir nommée constate-t-on l'émergence mais sans l'expliquer. Les renvois aux formulations de l'auto-organisation sont tout autant énigmatiques: rien dans le niveau "micro" (inférieur) ne permet de rendre compte de ce qui va apparaître, les caractéristiques du niveau "macro" (supérieur) ne sont pas contenues dans le niveau "micro".

E. Bernard-Weil se demande alors si la systémique ago-antagoniste dont il est le père ne permettrait pas de sortir de ce paradoxe en mettant à l'origine de toute forme vivante, y compris la plus simple comme la bactérie, la boucle ago-antagoniste excitation-inhibition déjà potentiellement présente dans les composants matériels organiques (protéines et $\mathrm{ADN}$ ) de la bactérie. On aurait là le fondement de l'extraordinaire créativité de l'évolution, fondement déjà présent lors de la naissance des protobactéries et se déployant ensuite par la multiplication des niveaux d'organisation lors du déploiement des vivants multi-cellulaires. 
Il convient de rappeler ici en quoi consiste la systémique ago-antagoniste de Bernard-Weil, dérivée de ses travaux en médecine hospitalière sur les maladies immunitaires ayant pour origine un dérèglement cellulaire. Il observait alors que le déséquilibre hormonal, cause du symptôme, pouvait parfois être efficacement combattu par l'administration non pas de l'hormone manquante (ce qui semble logique), mais de l'hormone déjà en excès, ce qui est tout à fait paradoxal ! Il en déduisait le concept d'homéostasie (ou autonomie) pathologique, signifiant par cela que l'organisme vivant est capable de trouver des formes stables d'équilibre en dehors de ce qui est considéré habituellement comme la normalité (ici la santé). Cet équilibre pathologique peut se représenter de manière dynamique sous la forme d'une boucle de rétroaction constituée par une double relation d'inhibition (de l'hormone A par l'hormone B) et d'activation (de l'hormone B par l'hormone A). A l'état habituel, cet équilibre est stable (boucle négative) bien que gravement pathologique (excès de $\mathrm{A}$, insuffisance de $\mathrm{B}$ ).

Les boucles de rétroaction présidant au fonctionnement de tels systèmes ont le plus souvent une polarité ambivalente, c'est à dire susceptible suivant la situation d'être négative (jouant en faveur de la stabilité et du retour à l'équilibre) ou positive (jouant en faveur du changement par accroissement des divergences ou "effet boule de neige"). De telles boucles, dites ago-antagonistes, sont légion dans les systèmes biologiques et plus encore dans les systèmes sociaux. Le systémicien représente cette causalité circulaire d'un type particulier de la manière suivante :

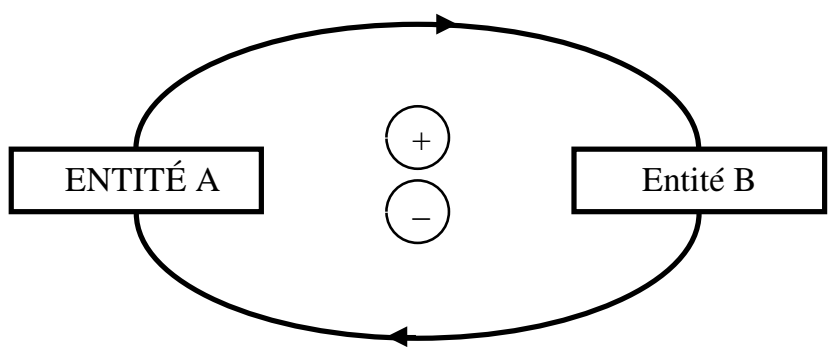

Dans une telle boucle, les deux pôles doivent être maintenus ensemble, dans la radicalité de leur opposition comme de leur coopération, car c'est de cette division constituante que le couple agoantagoniste tire son efficacité. Cette caractéristique interdit toute recherche de synthèse, de compromis, de voie moyenne, de juste milieu,... dans le jeu interactif des deux pôles.

Une illustration peut être donnée de cette caractéristique, empruntée au champ des sciences sociales où le concept trouve des applications nombreuses. Il s'agit de l'usage du diptyque féliciter/réprimander en matière éducative. La position moyenne consistant pour des parents, à dire "globalement ça peut aller" est tout à fait néfaste. Les enfants ont horreur de l'eau tiède et veulent que leurs parents leur disent clairement ce qu'ils apprécient et ce qui leur déplaît. Il faut donc utiliser à plein la félicitation comme la réprimande et non rechercher une position médiane. La félicitation tire sa valeur de la réprimande et vice-versa. Et un enfant n'est guère stimulé par un parent qui ne félicite jamais ou n'ose pas réprimander.

Pour Elie Bernard-Weil, c'est une "propriété fondamentale de l'être vivant que de comporter des couples de fonctions opposées quelque soit le niveau d'observation dans l'échelle des émergences". De tels couples ago-antagonistes peuvent jouer au sein d'un même niveau d'organisation ou entre niveaux d'organisation différents. Il s'agirait en quelque sorte d'une sorte de loi générale de la vie s'appliquant à tous les niveaux d'organisation et que l'on peut considérer comme "l'immergence d'un logos ago-antagoniste" dans les processus vitaux.

Flirtant alors avec la métaphysique, sinon la religion, Bernard-Weil en vient à considérer ce logos agoantagoniste comme l'invariant suprême de l'évolution, agissant sur elle de manière quasitranscendante. 


\section{LA THEORIE DE L'AUTONOMIE SELON LA PENSEE DE PIERRE VENDRYES}

(J. Lorigny, B. Paulre, R. Vallee, F. Le Gallou, E. Bernard-Weil, J. Fourastie, 1995 : vol.9, $\mathrm{n}^{\circ} 5$, pp.491-521)

Pierre Vendryès (1908-1989) est un médecin et philosophe français, passionné d'anthropologie et qui aura cherché, durant toute sa vie, à bâtir une théorie de l'homme en s'inspirant de la méthode expérimentale de Claude Bernard. Chemin faisant, il aura réussi à donner une définition extrêmement performante de ce qu'est le vivant, à formuler avant Norbert Wiener les premiers principes de la cybernétique, à relier de manière organique autonomie, causalité et probabilités. Ceci explique l'aura qu'il avait acquise auprès de nombreux chercheurs et pourquoi pas moins de huit personnes se sont mises en demeure de célébrer sa mémoire dans ce numéro de la RIS qui lui était en partie consacré à l'occasion du cinquième anniversaire de sa mort.

\section{Le concept d'autonomie chez le vivant : l'interprétation systémique de $P$. Vendryès}

L'énoncé fondamental de Vendryès "L'organisme, en acquérant son autonomie par rapport à son milieu extérieur et à partir de lui, acquiert la possibilité d'entrer avec lui en relations aléatoires" signifie tout simplement que l'être vivant peut échapper partiellement au déterminisme de son milieu, cela en imaginant des réponses qui lui sont propres et de soi imprévisibles (aléatoires) à partir des seules caractéristiques du milieu.

Cet indéterminisme relationnel, tel que défini par Vendryès, est une propriété inhérente du vivant et résulte de la constitution par l'organisme de réserves de matière, d'énergie et d'information, ainsi que de l'existence d'une "clôture organisationnelle" (la membrane cellulaire, l'exoderme d'un organisme vivant) qui sélectionne les échanges avec le milieu et ne subit pas passivement son influence.

Or, de telles caractéristiques du rapport organisme / milieu peuvent se représenter aisément au moyen de la boucle de rétroaction ago-antagoniste du systémicien. En traduisant le caractère interactif et aléatoire (grâce à sa bipolarité ou ago-antagonisme) du rapport de l'organisme à son milieu, cette boucle exprime parfaitement l'autonomie du vivant.

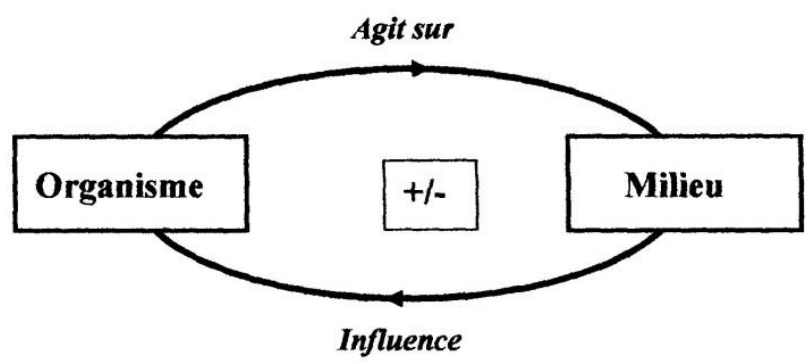

Si la boucle se réduit à la flèche du bas, on retrouve le déterminisme ordinaire des sciences physiques. En revanche, la réduction de la boucle à la flèche du haut traduirait l'existence d'un organisme tout puissant façonnant le milieu à sa convenance. En réalité, on se trouve toujours dans un mixte des deux, une interaction dynamique des deux pôles à caractère ago-antagoniste : la boucle peut fonctionner aussi bien sur un mode stabilisé (rétroaction dite négative) dans lequel organisme et milieu s'épaulent pour maintenir un équilibre stable, que sur un mode divergent (rétroaction dite positive) dans lequel organisme et milieu amplifient chacun de leur côté un changement. Comme l'a montré Stephen Jay Gould, l'histoire de la vie n'est pas avare d'exemples de cette nature en faisant alterner de longues périodes de stabilité (dites stases) avec de soudaines mutations. 


\section{La théorie de l'homme de $P$. Vendryès}

La question de la liberté humaine n'a pas cessé de hanter, depuis l'aube de l'humanité, les sagesses religieuses et philosophiques. Mes actions sont-elles vraiment les miennes et en ai-je la libre décision? Ou bien me sont-elles dictées par des puissances supérieures venues de l'extérieur (les dieux, le destin, les déterminismes sociaux) ou pire encore de mon propre organisme (les pulsions, les instincts), puissances dont je suis le jouet inconscient?

Les deux réponses ont toujours coexisté, mais selon des proportions variables, dans les traditions religieuses et dans les discours des philosophes. Tant que le débat pour ou contre la liberté humaine est resté au niveau des convictions religieuses ou philosophiques, il a pu sembler sans issue. C'est pourquoi il se poursuit depuis des millénaires sans que l'un des camps ait pu vraiment l'emporter. On remarquera d'ailleurs que la même position peut, suivant les lieux et les moments historiques, être tenue alternativement par les mêmes protagonistes (gratifiés pour la circonstance d'une étiquette droite ou gauche, conservateur ou progressiste, etc.) donnant naissance à d'étranges combats à front renversé.

Est-il alors possible par une connaissance d'intention scientifique, fondée sur l'observation et la modélisation, d'arracher ce débat à son enlisement ? Telle est bien l'ambition de Pierre Vendryès qui pense pouvoir le faire à partir de sa définition de l'autonomie chez le vivant. Qu'advient-il en effet lorsque le vivant devient aussi pensant? Tout se joue dans ce cas au niveau du système nerveux central avec le gros cerveau humain aux cent milliards de neurones interconnectés et dont ce que nous appelons le psychisme humain est la manifestation sociale visible. S'agit-il d'une simple caractéristique secondaire dans l'évolution de la vie, comme la patte coureuse de l'équidé, ou bien d'une différence de nature, d'un saut dans un nouvel ordre de réalité ? Il semble bien que Vendryès se prononce en faveur de cette seconde réponse à partir de la considération des stocks requis par cette autonomie. Pour le pensant, le stock en informations (notamment en informations symboliques) est considérablement supérieur à ce qu'il est pour le vivant ordinaire. Il y aurait bien là un changement de nature.

Cette réponse se trouve aujourd'hui renforcée par les travaux menés dans les sciences de la cognition. Je citerai ainsi la position de l'une des spécialistes du domaine, Evelyne Andreewsky, pour qui la cognition, c'est-à-dire l'esprit, est une propriété émergeant non seulement de la biologie (le système neuronal), mais aussi du langage humain, lequel est une réalité de nature symbolique et culturelle. Elle écrit :"L'esprit et la cognition constituent, par construction, un phénomène à la fois biologique, culturel et social, émergeant de l'intégration de toutes ces dimensions". En termes de représentation systémique, cela se traduit par la double boucle du schéma ci-après. Le psychisme humain est à la fois en interaction avec le système neuronal, enraciné dans le corps biologique et par voie de conséquence la biosphère, et avec le système symbolico-culturel du groupe social, expression locale de la noosphère. Et chacune de ces boucles est bien entendu ago-antagoniste.

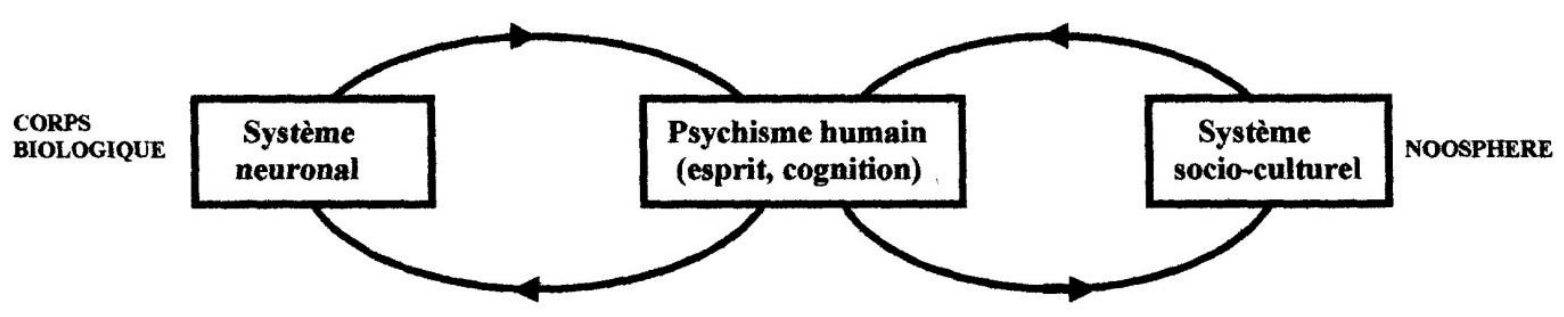

L'autonomie de l'être humain, à la différence de l'animal, se caractériserait donc par une relation doublement aléatoire, car en dépendance de deux milieux distincts : la biosphère et la noosphère. N'est-ce pas l'articulation de ces deux boucles aléatoires qui se trouve à l'origine de ce que nous appelons libre-arbitre ou liberté ? Pierre Vendryès avait repéré cette particularité lorsqu'il disait que pour fonder une anthropologie, il fallait englober de façon cohérente à la fois la réalité matérielle et le 
"penser" (c'est-à-dire l'esprit, la cognition, les représentations mentales). Pour lui, il s'agissait de deux univers aux causalités différentes dont toute vraie théorie de l'homme devait montrer l'articulation ${ }^{1}$.

Pour rendre compte de cette articulation, les neuro-sciences comme la psychologie expérimentale ne nous offrent pour le moment que des connaissances analytiques et parcellaires. Elles débouchent sur des bribes de théorie, intéressantes certes mais bien incapables de fournir les matériaux pour construire un modèle global et intégré du psychisme humain. A vrai dire, les seules ressources disponibles pour un tel projet sont aujourd'hui à rechercher du côté de la psychanalyse.

Or, l'apport de la psychanalyse va se présenter d'entrée de jeu comme ambigu et paradoxal. Dans un premier temps, la psychanalyse semble apporter des arguments en faveur du déterminisme. En dévoilant les variables cachées de l'inconscient, la psychanalyse montre que la conscience, le sujet, la liberté ne sont pas ce qu'ils croient être. Mais lorsque l'on pousse plus loin la modélisation, on voit alors apparaître des bifurcations, de l'aléatoire, de l'imprévisible d'où peut émerger la flamme fragile d'une liberté.

\title{
4. LA SYSTEMIQUE : UN META-LANGAGE CONNECTIF PAR CHARLES FRANÇOIS
}

\author{
(1998 : vol.12, n4-5, pp.405-418)
}

écrivant son article en 1998, alors que la systémique peut se prévaloir en Europe d'une pratique de plus de 30 années, Charles François croit souhaitable et possible de faire un point de situation de cette nouvelle discipline du savoir, d'en rappeler l'histoire, d'en présenter les débats en cours, d'en induire une perspective de développement pour le futur.

\section{Comment la systémique, fille de la cybernétique, est devenue un nouveau discours de la méthode}

C. François rappelle d'abord comment à partit des concepts issus de la cybernétique de la fin des années 1940, la systémique a dû :

- préciser la notion de système en indiquant les objets du monde qui en faisaient partie,

- ce qu'étaient les parties d'un système,

- qu'elles étaient les relations entre les parties

- qu'elles étaient les fonctions remplies par ces différentes parties

- comment s'établissait et se maintenait la cohérence du système

- quelles relations entre le système et son environnement.

Chemin faisant, à partir d'autres concepts empruntés à d'autres disciplines comme la thermodynamique, la biologie, les mathématiques, la linguistique, l'anthropologie, etc., la systémique a dû sortir de l'ambiguité système ouvert/système fermé en précisant ce qu'est la notion d'ouverture et celle de frontière. Tout système complexe est sélectivement ouvert ou fermé selon la nature des intrants et des extrants dont il dépend pour son existence (flux énergétiques, matériels et informatifs). D'où des questions nouvelles concernant la sélection des intrants et les conditions d'environnement favorables pour le système.

\footnotetext{
${ }^{1}$ On observera que Pierre Vendryès retrouve ici la théorie des trois mondes de l'épistémologue Karl POPPER : celui-ci distingue le monde des objets réels (le premier monde), le monde des états mentaux (le deuxième monde) et utilise l'expression "troisième monde" pour désigner les "contenus objectifs de pensée" (ou représentations) issus du système culturel.
} 
Vint ensuite, à partir des travaux de H. Simon sur les systèmes artificiels, la question des niveaux d'organisation dans les systèmes et les premières tentatives de typologie pouvant s'appliquer, suivant le cas, aux systèmes vivants, aux systèmes artificiels et aux systèmes sociaux.

\section{Les questions en débat}

Une connexion serait à établir, en termes de théorie de la régulation, avec des travaux originaux mais restés un peu en marge comme ceux de P. Vendryès (sur l'autonomie), de R. Ashby (sur la variété requise), de Maturana (sur l'autopoièse). De manière générale, un programme de recherche de caractère transdisciplinaire pourrait être développé en direction, notamment, de divers systèmes de l'environnement dotés de propriétés singulières qui ont déjà pu être étudiées séparément.

Ainsi des régulations écologiques, d'accès et de localisation souvent difficiles et pour cette raison souvent mal comprises et manipulées à mauvais escient. De même le lien à établir avec la théorie mathématique des fractales de Benoît Mandelbrot, laquelle montre une similitude de forme par-delà les niveaux d'organisation. Ainsi également avec la théorie des attracteurs chaotiques et celle des catastrophes de René Thom régissant les espaces topologiques.

On le voit, de ce point de vue, la systémique est encore une discipline toute jeune qui semble appelée à de vastes développements.

\section{Un méta-langage pour les sciences?}

La systémique apparait donc comme une collection de modèles et de concepts extrêmement généraux, chacun doté d'une grande capacité analogique, analogie qui se trouve ainsi largement réhabilitée dans la méthode scientifique. Utilisé en effet par les philosophes de l'Antiquité et les théologiens médiévaux, ce mode de raisonnement s'est trouvé décrié au $19^{\text {ème }}$ siècle au nom d'un rationalisme étroit...alors même qu'il continuait d'imprégner la démarche heuristique des chercheurs. Ces derniers n'hésitaient pas à transposer de manière "sauvage" des concepts venus d'autres disciplines, quitte à le justifier ensuite par de laborieux raisonnements déductifs. Comme le souligne Edgar Morin":"La circulation clandestine des concepts a permis aux disciplines de se désasphyxier".

En matière d'analogie, trois niveaux peuvent être distingués :

- la métaphore établit une correspondance souvent toute extérieure entre deux séries de phénomènes différents ou deux systèmes de nature différente. Parce qu'elle se fonde sur l'apparence, la métaphore est dangereuse et tombe sous les coups de l'adage positiviste "comparaison n'est pas raison". Bien utilisée, la métaphore est néanmoins précieuse. En stimulant l'imagination, elle facilite la création de nouveaux modèles et constitue bien souvent un premier pas vers l'homomorphisme,

- l'homomorphisme établit une correspondance surjective entre quelques traits du système étudié et les traits d'un modèle théorique ou d'un système concret plus simple ou plus commodément étudiable (que l'on appelle alors modèle réduit). Par des observations effectuées sur le second système, il devient possible de prévoir certains aspects du comportement du premier.

- l'isomorphisme est la seule analogie acceptable dans une démarche analytique de type cartésien. Il s'agit d'établir une correspondance bijective entre tous les traits de l'objet étudié et ceux d'un modèle censé reproduire une image de l'original où rien n'est oublié, ni dans la description, ni dans le fonctionnement.

Utilisable pour les systèmes à faible complexité de la physique et de la chimie, l'isomorphisme n'est guère tenable pour les systèmes complexes, et cela malgré la tentation d'augmenter considérablement les variables prises en compte. Par un glissement inévitable, on en est donc venu à accepter l'imperfection du modèle comme un mal nécessaire et donc à privilégier l'homomorphisme.

Le retournement de perspective apporté alors par l'approche systémique consiste à considérer que cette analogie honteuse, tout juste bonne selon certains pour les sciences humaines, est en réalité la

\footnotetext{
${ }^{2}$ MORIN E. : Introduction à la pensée complexe, p. 154, ESF 1991
} 
condition nécessaire de toute connaissance. Le modèle homomorphe est sans doute plus simple que le réel, mais c'est pourquoi nous le comprenons, pourquoi nous pouvons l'utiliser pour orienter nos actions. Comme le note Claude Lévi-Strauss ${ }^{3}$. "La vertu intrinsèque du modèle réduit est qu'il compense la renonciation à des dimensions sensibles par l'acquisition de dimensions intelligibles". Et ceci est particulièrement heureux car le but d'un modèle n'est pas l'exhaustivité. Il est des détails inutiles pour le but visé, que ce but soit la construction de modèles pour comprendre (qui doivent être suffisamment simples) ou de modèles pour agir (qui doivent être efficaces).

A ce stade, la systémique pourrait apparaître comme la science générale des modèles. "Et son efficacité maxima ne se manifestera, écrit Charles François, que lorsqu'elle deviendra réellement un langage cohérent, articulé, non contradictoire qui se répandra dans tous les domaines de la pensée, de la recherche et de l'action." Ainsi la systémique deviendrait-elle ce nouveau discours de la méthode pour la science, ou encore cet outil privilégié pour observer le réel et que Joël de Rosnay appelle le macrocospe, ou bien encore cette nouvelle approche pour VOIR la plénitude du Réel et que Teilhard de Chardin appelle sa phénoménologie.

\footnotetext{
${ }^{3}$ LEVI-STRAUSS C. : La pensée sauvage, Plon 1962
} 